\title{
ADAPTIVE MESH RECONSTRUCTION FOR HYPERBOLIC CONSERVATION LAWS WITH TOTAL VARIATION BOUND
}

\author{
NIKOLAOS SFAKIANAKIS
}

\begin{abstract}
We consider 3-point numerical schemes, that resolve scalar conservation laws, that are oscillatory either to their dispersive or anti-diffusive nature. The spatial discretization is performed over non-uniform adaptively redefined meshes. We provide a model for studying the evolution of the extremes of the oscillations. We prove that proper mesh reconstruction is able to control the oscillations; we provide bounds for the Total Variation (TV) of the numerical solution. We, moreover, prove under more strict assumptions that the increase of the TV, due to the oscillatory behavior of the numerical schemes, decreases with time; hence proving that the overall scheme is TV Increase-Decreasing (TVI-D). We finally provide numerical evidence supporting the analytical results that exhibit the stabilization properties of the mesh adaptation technique.
\end{abstract}

\section{Outline}

Mesh adaptation techniques have been employed by several authors in the past. It is worth noting the seminal works [DD87, For88, HH83, Luc85, Luc86, TT03, where several properties of mesh adaptation were studied. It was noticed in AKM01 AMT04, AMS10, Sfa09, that proper use of non-uniform adaptively redefined meshes is capable of taming oscillations; hence improving the stability properties of the numerical schemes.

To study the stabilization properties of mesh adaptation techniques we analyze the effect they have, on the oscillations that oscillatory/unstable numerical schemes produce. The setting is the one-dimensional scalar Riemann problem: $u_{t}+f(u)_{x}=$ $0, x \in[a, b]$, with the flux function $f$ being smooth and convex. For initial conditions we consider the single jump $u_{0}(x)=\mathcal{X}_{\left[0, x_{0}\right]}(x)$ with $x, x_{0} \in(0,1)$.

We discretize spatially over a non-uniform adaptively redefined mesh. The mesh adaptation and the time evolution of the numerical solution are combined into the Main Adaptive Scheme:

Definition 1.1 (Main Adaptive Scheme (MAS)). Given, at time step $n$, the mesh $M_{x}^{n}=\left\{a=x_{1}^{n}<\cdots<x_{N}^{n}=b\right\}$ and the approximations $U^{n}=\left\{u_{1}^{n}, \ldots, u_{N}^{n}\right\}$, the steps of the (MAS) are:

1. (Mesh Reconstruction). Construct new mesh: $M_{x}^{n+1}=\left\{a=x_{1}^{n+1}<\cdots<\right.$ $\left.x_{N}^{n+1}=b\right\}$.

2. (Solution Update). Use the old mesh $M_{x}^{n}$ the approximations $U^{n}$ and the new mesh $M_{x}^{n+1}$ :

Received by the editor September 19, 2009 and in revised form, September 2, 2011.

2010 Mathematics Subject Classification. Primary 65-XX.

(C) 2012 American Mathematical Society 
2a. to construct a piecewise linear function $V^{n}(x)$ such that $\left.V^{n}\right|_{M_{x}^{n}}=U^{n}$ and,

2b. define the updated approximations $\hat{U}^{n}=\left\{\hat{u}_{i}^{n}, \ldots, \hat{u}_{N}^{n}\right\}$ as $\hat{U}^{n}=$ $\left.V^{n}\right|_{M_{x}^{n+1}}$.

3. (Time Evolution). Use the new mesh $M_{x}^{n+1}$, the new approximations $\hat{U}^{n}$ and the numerical scheme to march in time and compute $U^{n+1}=$ $\left\{u_{1}^{n+1}, \ldots, u_{N}^{n+1}\right\}$.

The main objective of this work is to place conditions on the steps of MAS such that the resulting numerical solutions are TV stable even when oscillatory numerical schemes are used for the time evolution (Step 3). More specifically, we prove that proper non-uniform meshes are able to tame the TV increase due to oscillations; furthermore, we prove that in some cases the TV increase due to oscillation decreases with time, hence yielding a Total Variation Increase Diminishing (TVID) scheme.

In section 2 of this work we list and explain the requirements that we place on the MAS. In section 3. we discuss the creation and propagation of oscillations at the level of extremes. We present a model for the extremes that takes into account the steps of MAS. Based then on the model we prove the TV result of this work Theorem 3.1. In section 4 we discuss the Main Adaptive Scheme (MAS) in more detail. We analyse the way non-uniform meshes are constructed and how the numerical solution is updated over the new mesh. We discuss the numerical implementation of the MAS and present some graphs depicting its basic properties. In section 5 we discuss the numerical implementation of the requirements introduced in section 2, In section 6 we perform numerical tests, where we consider known oscillatory numerical schemes and prove that these schemes satisfy the requirements introduced in section 2, We provide comparative numerical results between uniform and non-uniform mesh cases, where we observe the stabilization properties of the the mesh reconstruction.

\section{REQUiREMENTS}

In this section we present the requirements that we place on the steps of MAS. For the Solution Update (Step 2), we perform interpolation over piecewise linear functions and for the Time Evolution (Step 3) we use oscillatory Finite Difference schemes. The proper setting for dealing with Finite Volume schemes with a conservative reconstruction will be presented separately.

Let $M_{x}^{n}=\left\{x_{i}^{n}, i=1, \ldots, N\right\}$ be the initial mesh, $U^{n}=\left\{u_{1}^{n}, \ldots, u_{N}^{n}\right\}$ the initial approximations at the time step $n$, and let $M_{x}^{n+1}=\left\{x_{j}^{n+1}, j=1, \ldots, N\right\}, \hat{U}^{n}=$ $\left\{\hat{u}_{1}^{n}, \ldots, \hat{u}_{N}^{n}\right\}$ be the new mesh and updated approximations yielding from Steps 1 and 2 of the MAS.

The discussion that will take place and the proofs that will be presented are valid for every numerical scheme that satisfies the Evolution requirement:

Requirement 1 (Evolution requirement). There exists a constant $C>0$ independent of the time step $n$ and the node $i$ such that:

$$
\left|u_{i}^{n+1}-\hat{u}_{i}^{n}\right| \leq C \max \left\{\left|\hat{u}_{i+1}^{n}-\hat{u}_{i}^{n}\right|,\left|\hat{u}_{i}^{n}-\hat{u}_{i-1}^{n}\right|\right\} .
$$

Remark 1. In the examples addressed in section 6, we see that the constant $C$ is an increasing function of the CFL condition. For every scheme we use, we prove 


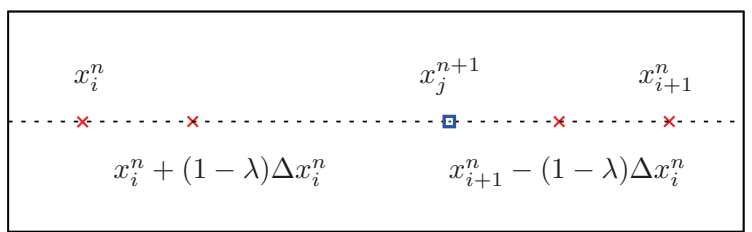

Figure 1 . The $\lambda$-rule states that the new node $x_{j}^{n+1} \in\left[x_{i}^{n}, x_{i+1}^{n}\right]$, and avoids the old node $x_{i}^{n}$ (respectively $x_{i+1}^{n}$ ), where the approximate solution might exhibit an extreme, in the sense that $x_{i}^{n}+(1-\lambda) \Delta x_{i}^{n} \leq x_{j}^{n+1}$ (respectively $\left.x_{j}^{n+1} \leq x_{i+1}^{n}-(1-\lambda) \Delta x_{i}^{n}\right)$, where $\Delta x_{i}^{n}=x_{i+1}^{n}-x_{i}^{n}$.

this requirement and also compute the dependence of the constant $C$ to the CFL condition.

We move on to the second requirement, which is placed on the mesh reconstruction procedure (Step 1). We first start with a definition.

Definition 2.1. We say that the approximate solution $U^{n}=\left\{u_{i}^{n}, i=1, \ldots, N\right\}$ defined over the mesh $M_{x}^{n}=\left\{x_{i}^{n}, i=1, \ldots, N\right\}$ exhibits a local extreme at the node $x_{i}^{n}$ if $u_{i}^{n}>u_{i-1}^{n}, u_{i+1}^{n}$ (local maximum) or $u_{i}^{n}<u_{i-1}^{n}, u_{i+1}^{n}$ (local minimum).

Requirement 2 (Mesh requirement: $\lambda$-rule). There exists a constant $0<\lambda<1$ independent of $n$ and $i$ such that, if $x_{j}^{n+1} \in\left[x_{i}^{n}, x_{i+1}^{n}\right]$ and $U^{n}$ exhibits a local extreme at the node $i$ (resp. $i+1)$, then

$x_{j}^{n+1}-x_{i}^{n}>(1-\lambda)\left(x_{i+1}^{n}-x_{i}^{n}\right)$ $\left(\right.$ resp. $\left.x_{i+1}^{n}-x_{j}^{n+1}>(1-\lambda)\left(x_{i+1}^{n}-x_{i}^{n}\right)\right)$.

Remark 2. The meaning of the $\lambda$-rule requirement (2), is that the new nodes $x_{j}^{n+1}$ avoid the places of the old extremes $x_{i}^{n}\left(\right.$ or $\left.x_{i+1}^{n}\right)$ by at least $1-\lambda$ of the respective interval $\left(x_{i}^{n}, x_{i+1}^{n}\right)$.

The $\lambda$-rule requirement is placed on the mesh reconstruction but also affects the values of piecewise linear functions. The following remark discusses their relation.

Remark 3 ( $\lambda$-rule effect for piecewise linears and interpolation). We assume that $u(x)$ is a piecewise linear function that oscillates as depicted in Figure 2 We assume, moreover, that the new nodes respect the $\lambda$-rule requirement (2) at the extremes in the respective subintervals and that $y=v$ is a horizontal line that separates the extremes.

Accordingly, $x_{j-1}^{n+1} \in\left[x_{i-1}^{n}, x_{i}^{n}\right]$ and by the $\lambda$-rule requirement we get:

$$
\frac{x_{i}^{n}-x_{j-1}^{n+1}}{x_{i}^{n}-x_{i-1}^{n}} \leq \lambda .
$$

The linearity and monotonicity of $u$ in $\left[x_{i-1}^{n}, x_{i}^{n}\right]$ recast the previous into:

$$
u\left(x_{j-1}^{n+1}\right)-u\left(x_{i}^{n}\right) \leq \lambda\left(u\left(x_{i-1}^{n}\right)-u\left(x_{i}^{n}\right)\right) .
$$

Since $0<\lambda<1$ and $u\left(x_{i}^{n}\right)<v$ the previous reads:

$$
u\left(x_{j-1}^{n+1}\right) \leq \lambda u\left(x_{i-1}^{n}\right)+(1-\lambda) u\left(x_{i}^{n}\right) \leq \lambda u\left(x_{i-1}^{n}\right)+(1-\lambda) v
$$




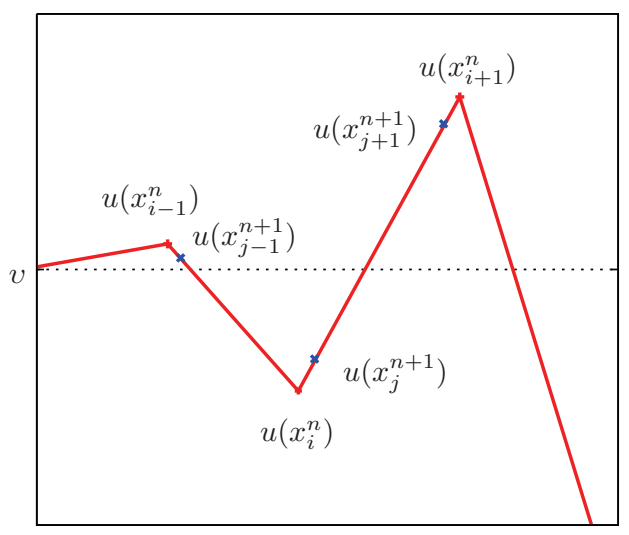

Figure 2. This figure depicts the application of the $\lambda$-rule in the case of a piecewise linear function. The places of the new nodes are depicted along with the old extremes.

or

$$
u\left(x_{j-1}^{n+1}\right)-v \leq \lambda\left(u\left(x_{i-1}^{n}\right)-v\right) .
$$

Passing to the third requirement, we first note that the overall phenomenon consists of two major steps, the mesh reconstruction (Step 1) and the time evolution (Step 3). We need to study these steps both separately and together. For the separate analysis the requirements (11) and (2) are sufficient, but for the joined analysis one more requirement is needed. This is due to the fact that (Step 1) cannot be analyzed with classical methods (such as the modified equation of the scheme) since it takes place between two consequent time steps, i.e., the mesh reconstruction is not related to the time evolution of the numerical scheme.

The merging of these steps is quantified in the Coupling requirement, and constitutes the major contribution of this work:

Requirement 3 (Coupling requirement). The constants $C$ of the evolution requirement (11) and $\lambda$ of the $\lambda$-rule requirement (2) are connected via the following relation:

$$
\lambda+3 \lambda C<1 .
$$

\section{TIME STEP ANALYSIS}

In this section we discuss the appearance and evolution of local extremes. We devise recursive, with respect to the time step $n$, relations regarding the evolution of the extremes. We present and prove the theoretical results of this work; these include bounds on the extremes, bounds on the TV increase due to oscillations, moreover, we prove that in some cases the TV increase, decreases with time steps $n$.

3.1. Recursive relations. The discussion regarding the creation and evolution of the extremes is performed in a per time step manner. In every time step we discuss their temporal evolution and their spatial modification.

In the description that follows we split every step into two sub-steps. The first sub-step is the time evolution which is due to the numerical scheme and is governed 


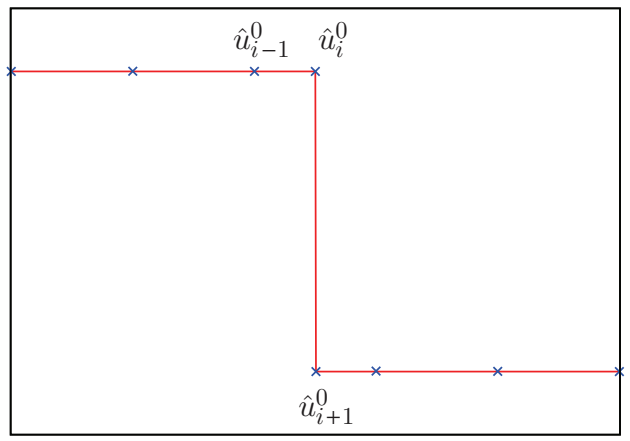

Figure 3. This is the initial condition. In this configuration we set $\hat{a}_{1}=\left|\hat{u}_{i}^{0}-\hat{u}_{i+1}^{0}\right|$

by the evolution requirement (11), and the second sub-step is the spatial modification which is due to the mesh relocation and the solution update procedure and is governed by the $\lambda$-rule requirement (2) and the coupling requirement (3).

1st step. We refer to Figure 3 for a graphical description of the initial configuration. The first nodal point, $\hat{u}_{i}^{0}$, located at the top of the shock, that is, will evolve according to the evolution requirement (11):

$$
\left|u_{i}^{1}-\hat{u}_{i}^{0}\right| \leq C \max \left\{\left|\hat{u}_{i}^{0}-\hat{u}_{i-1}^{0}\right|,\left|\hat{u}_{i}^{0}-\hat{u}_{i+1}^{0}\right|\right\} .
$$

We consider jump initial conditions; hence $\left|\hat{u}_{i}^{0}-\hat{u}_{i-1}^{0}\right|=0$ and $\left|\hat{u}_{i}^{0}-\hat{u}_{i+1}^{0}\right| \leq T V\left(u^{0}\right)$. We denote $\hat{a}_{1}=\left|\hat{u}_{i}^{0}-\hat{u}_{i+1}^{0}\right|$, and $a_{1}=C \hat{a}_{1}$, so the evolution requirement (10) reads:

$$
\left|u_{i}^{1}-\hat{u}_{i}^{0}\right| \leq C \max \left\{\left|\hat{u}_{i}^{0}-\hat{u}_{i-1}^{0}\right|,\left|\hat{u}_{i}^{0}-\hat{u}_{i+1}^{0}\right|\right\} \leq C\left|\hat{u}_{i}^{0}-\hat{u}_{i+1}^{0}\right|=C \hat{a}_{1}=a_{1} .
$$

To introduce the notation, we set $E_{1}^{1 / 2}$ to be the maximum magnitude of this extreme:

$$
E_{1}^{1 / 2}=\left|u_{i}^{1}-\hat{u}_{i}^{0}\right|=a_{1} .
$$

We refer to Figure 4 for a graphical description. We then perform the mesh reconstruction step and because of the $\lambda$-rule requirement (2) (see also Remark 31) the new extreme will be of magnitude bounded by

$$
E_{1}^{1}=\lambda a_{1},
$$

where full superscript ${ }^{1}$ is used since the relocation has taken place.

Remark 4. ( $E_{m}^{k}$ Notation) We denote by $E_{m}^{k+1 / 2}$ (half superscript) the bound on the magnitude of the $m$ th extreme at the $k$ th time step after time evolution and before the mesh reconstruction procedure. We denote by $E_{m}^{k+1}$ (full superscript) the bound on the magnitude of $m$ th extreme at the end of the $k$ th time step, that is after the time evolution and the mesh reconstruction procedure.

2nd step. We refer Figure 4 for the situation at the end of the 1st step, where we had one extreme of magnitude bounded by $E_{1}^{1}=\lambda a_{1}$. Due to the time evolution we expect the 1st extreme to evolve to a new value and also the creation of a 2 nd extreme at the left side of the 1st extreme. We will study each extreme separately. 


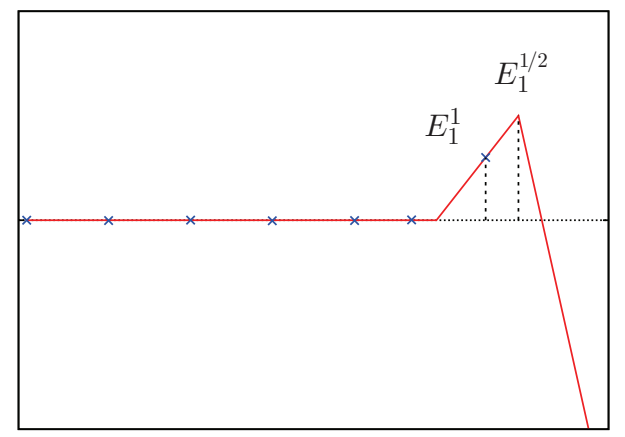

FIgURE 4. The situation at the head of the shock at the end of the 1-st time step. Only one extreme exists and is of magnitude $E_{1}^{1}$. The numerical solution before the remeshing procedure is depicted with the continuous line, and the new nodes that occur after the remeshing procedure are depicted with points.

1st Extreme. The evolution requirement (1) dictates that this extreme shall evolve as:

$$
\left|u_{i}^{1+1 / 2}-\hat{u}_{i}^{1}\right| \leq C \max \left\{\left|\hat{u}_{i}^{1}-\hat{u}_{i-1}^{1}\right|,\left|\hat{u}_{i}^{1}-\hat{u}_{i+1}^{1}\right|\right\} .
$$

From the previous time step we have that $\left|\hat{u}_{i}^{1}-\hat{u}_{i-1}^{1}\right| \leq E_{1}^{1}$ and $\left|\hat{u}_{i}^{1}-\hat{u}_{i+1}^{1}\right| \leq$ $2 E_{1}^{1}+\hat{a}_{2}$. To justify the second inequality we return at the end of the time step $k=1$ and notice that the node $i+1$ is placed along the shock, which is, by symmetry, of variation at most $E_{1}^{1}+T V\left(u^{0}\right)+E_{1}^{1}$. So the evolution requirement (11) for the 1st Extreme reads:

$$
\left|u_{i}^{1+1 / 2}-\hat{u}_{i}^{1}\right| \leq C\left(2 E_{1}^{1}+\hat{a}_{2}\right)=2 C E_{1}^{1}+a_{2},
$$

where we have defined $a_{2}=C \hat{a}_{2}$. Now, if we set $v$ to be the level from which we measure the magnitudes of the extremes (in this case the top of the shock), the previous bound reads:

$$
\left|\left(u_{i}^{1+1 / 2}-v\right)-\left(u_{i}^{1}-v\right)\right| \leq 2 C E_{1}^{1}+a_{2} .
$$

By setting $E_{1}^{1+1 / 2}=u_{i}^{1+1 / 2}-v$ and since $E_{1}^{1}=u_{i}^{1}-v$ we deduce that the magnitude of the 1st extreme will be bounded as:

$$
E_{1}^{1+1 / 2} \leq E_{1}^{1}+2 C E_{1}^{1}+a_{2} .
$$

Now the relocation procedure takes place, and due of the $\lambda$-rule requirement (2), the magnitude of the 1st extreme will be bounded by

$$
E_{1}^{2}=\lambda\left(E_{1}^{1}+2 C E_{1}^{1}+a_{2}\right) .
$$

2nd Extreme. The evolution requirement (1) dictates that this extreme shall evolve as:

$$
\left|u_{i-1}^{1+1 / 2}-\hat{u}_{i-1}^{1}\right| \leq C \max \left\{\left|\hat{u}_{i-1}^{1}-\hat{u}_{i-2}^{1}\right|,\left|\hat{u}_{i-1}^{1}-\hat{u}_{i}^{1}\right|\right\} .
$$

From the previous time step we know that $\left|\hat{u}_{i-1}^{1}-\hat{u}_{i-2}^{1}\right|=0$ and $\left|\hat{u}_{i-1}^{1}-\hat{u}_{i}^{1}\right| \leq$ $E_{1}^{1}$. So the evolution requirement (1) recasts for the 2 nd extreme,

$$
\left|u_{i-1}^{1+1 / 2}-\hat{u}_{i-1}^{1}\right| \leq C E_{1}^{1},
$$




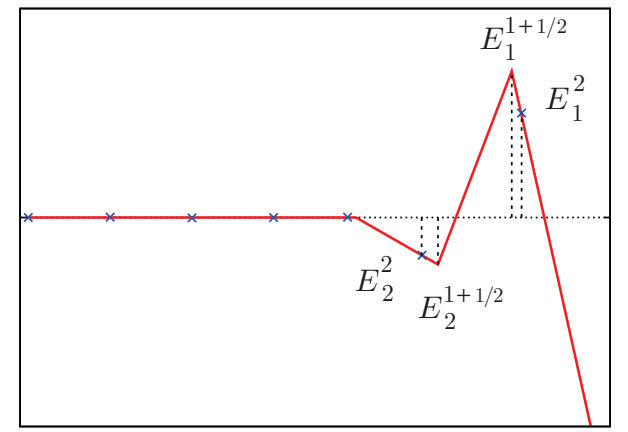

Figure 5. The resulting situation at the end of the 2nd time step. Two extremes of magnitudes $E_{1}^{2}$ and $E_{2}^{2}$ exist in this time step. The numerical solution before the remeshing procedure is depicted with the continuous line, and the new nodes that occur after the remeshing procedure are depicted with points.

or by noting that $u_{i-1}^{1}=v$ is the level from which we measure the magnitudes of the extremes, the previous bound recasts into

$$
E_{2}^{1+1 / 2} \leq C E_{1}^{1} \text {. }
$$

Now the relocation procedure takes place and the $\lambda$-rule requirement (2) dictates that the magnitude of the 2nd extreme at the end of this step shall be bounded by

$$
E_{2}^{2}=\lambda C E_{1}^{1} \text {. }
$$

So at the end of the 2nd step the bounds on the existing extremes are as follows:

$$
E_{1}^{2}=\lambda\left(E_{1}^{1}+2 C E_{1}^{1}+a_{2}\right), \quad E_{2}^{2}=\lambda C E_{1}^{1} .
$$

The situation at the end of this step is depicted in Figure (5).

3rd step. At the end of the previous step we had two extremes with magnitudes bounded by $E_{1}^{2}$ and $E_{2}^{2}$; see Figure (5). In this step we expect them to evolve to new values $E_{1}^{3}$ and $E_{2}^{3}$, we also expect a new extreme to appear, namely $E_{3}^{3}$. Arguing as previously, at the end of the 3rd step the bounds on the magnitudes of the extremes are (see Figure (6) ):

$$
\begin{aligned}
& E_{1}^{3}=\lambda\left(E_{1}^{2}+2 C E_{1}^{2}+a_{3}\right)=\lambda^{3}(1+2 C)^{2} a_{1}+\lambda^{2}(1+2 C) a_{2}+\lambda a_{3}, \\
& E_{2}^{3}=\lambda\left(E_{2}^{2}+C\left(E_{2}^{2}+E_{1}^{2}\right)\right)=\lambda^{3} 2 C(1+2 C) a_{1}+\lambda^{2} C a_{2}, \\
& E_{3}^{3}=\lambda C E_{2}^{2}=\lambda^{3} C^{2} a_{1} .
\end{aligned}
$$

For completeness we define the increases $a_{i}$. The variation of the shock consists of: a) the oscillatory part on the top, with magnitude at most $E_{1}^{k}$, b) the main part, with variation $T V\left(u^{0}\right)$, and c) the oscillatory part at the foot, with magnitude at most $E_{1}^{k}$.

Definition 3.1 ( $a_{i}$ increases). Let $\hat{u}_{i}^{k}$ be the value at the top of the shock. We define

$$
\hat{a}_{k}=\left(\left|\hat{u}_{i}^{k}-\hat{u}_{i+1}^{k}\right|-2 E_{1}^{k}\right)_{+},
$$

where the subscript + denotes the positive part. Moreover, we define $a_{k}=C \hat{a}_{k}$. 


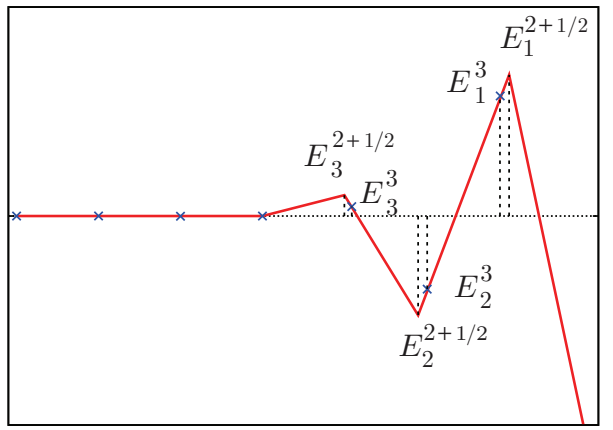

Figure 6. The resulting situation at the end of the 3rd time step. Three extremes of magnitude $E_{1}^{3}, E_{2}^{3}, E_{3}^{3}$ exist in this time step.

By definition, $\hat{a}_{k}$ describes the possibly more that $2 E_{1}^{k}$ distance $\left|\hat{u}_{i}^{k}-\hat{u}_{i+1}^{k}\right|$. That is, if $\left|\hat{u}_{i}^{k}-\hat{u}_{i+1}^{k}\right|<2 E_{1}^{k}$, then $\hat{a}_{k}=0$; hence $a_{k}=0$.

kth step. We generalize the previous discussion for the $k$ th time step and note that recursive relations for the extreme $m \leq k$ are given by

$$
\begin{cases}E_{m}^{k}=\lambda\left(E_{m}^{k-1}+C \cdot\left(E_{m}^{k-1}+E_{m-1}^{k-1}\right)\right), & \text { for } m>1, \\ E_{1}^{k}=\lambda\left(E_{1}^{k-1}+2 C \cdot E_{1}^{k-1}+a_{k}\right), & \text { for } m=1\end{cases}
$$

It is evident, from the second equation that we add at least $2 C E_{1}^{k-1}$ in the increase of the 1st extreme. This increases the magnitude of the 1st extreme but at the same time simplifies the presentation and the route of the proof.

In analysing these recursive relations, we see that for the evolution of the extreme $m$, from $E_{m}^{k-1}$ to $E_{m}^{k}$, we take into account the neighbouring extreme in the right hand side, $E_{m-1}^{k-1}$. To justify this choice, we easily prove by induction that the bounds $E_{m}^{k}$ on the magnitudes of the extremes constitute a decreasing sequence with respect to $m=1, \ldots$ for every step $k$, i.e.:

Lemma 3.1. For every step $k$ the magnitudes of the bounds given by (41) are in a decreasing order with respect to $m$.

3.2. Extremes. In this paragraph we solve the recursive relation (4), for every extreme $m$ and we provide uniform, with respect to the time step $k$, bounds on the magnitude of the extremes.

Lemma 3.2 (Magnitude of the 1st extreme). The magnitude of the first extreme in the kth time step is bounded by

$$
E_{1}^{k} \leq \lambda \sum_{j=1}^{k} \lambda^{k-j}(1+2 C)^{k-j} a_{j} \stackrel{l=k-j}{=} \lambda \sum_{l=0}^{k-1} \lambda^{l}(1+2 C)^{l} a_{k-l} .
$$


Proof. By induction. For the induction initiation and hypothesis we have:

$$
\begin{aligned}
& E_{1}^{1} \leq \lambda a_{1}=\lambda \sum_{j=1}^{1} \lambda^{1-j}(1+2 C)^{1-j} a_{j} \\
& E_{1}^{k} \leq \lambda \sum_{l=0}^{k-1} \lambda^{l}(1+2 C)^{l} a_{k-l} .
\end{aligned}
$$

For the induction step, the evolution relations (4) yield:

$$
\begin{aligned}
E_{1}^{k+1} & \leq \lambda\left(E_{1}^{k}+2 C E_{1}^{k}+a_{k+1}\right) \\
& \leq \lambda\left((1+2 C) \lambda \sum_{j=1}^{k} \lambda^{k-j}(1+2 C)^{k-j} a_{j}+a_{k+1}\right) \\
& \leq \lambda\left(\sum_{j=1}^{k} \lambda^{k+1-j}(1+2 C)^{k+1-j} a_{j}+\lambda^{k+1-(k+1)}(1+2 C)^{k+1-(k+1)} a_{k+1}\right) \\
& \leq \lambda \sum_{j=1}^{k+1} \lambda^{k+1-j}(1+2 C)^{k+1-j} a_{j} .
\end{aligned}
$$

Lemma 3.3 (Magnitude of the 2nd extreme). The magnitude of the second extreme in the kth time step is bounded by

$$
\begin{gathered}
E_{2}^{k} \leq \lambda^{2} C \sum_{j=1}^{k-1}\left(\begin{array}{c}
k-j \\
k-j-1
\end{array}\right) \lambda^{k-j-1}(1+2 C)^{k-j-1} a_{j} \\
\stackrel{l=k-j}{=} \lambda^{2} C \sum_{l=1}^{k-1}\left(\begin{array}{c}
l \\
l-1
\end{array}\right) \lambda^{l-1}(1+2 C)^{l-1} a_{k-l} .
\end{gathered}
$$

Proof. By induction. From (44), (5), (6) we get that

$$
\begin{aligned}
& E_{2}^{2} \leq \lambda^{2} C a_{1}=\lambda^{2} C \sum_{j=1}^{2-1}\left(\begin{array}{c}
2-j \\
2-j-1
\end{array}\right) \lambda^{2-j-1}(1+2 C)^{2-j-1} a_{j}, \\
& E_{2}^{k} \leq \lambda^{2} C \sum_{j=1}^{k-1}\left(\begin{array}{c}
k-j \\
k-j-1
\end{array}\right) \lambda^{k-j-1}(1+2 C)^{k-j-1} a_{j}
\end{aligned}
$$

and for the induction step we have

$$
\begin{aligned}
E_{2}^{k+1} & =\lambda\left(E_{2}^{k}+C\left(E_{2}^{k}+E_{1}^{k}\right)\right) \\
& \leq \lambda\left(\lambda^{2} C(1+C) \sum_{l=1}^{k-1}\left(\begin{array}{c}
l \\
l-1
\end{array}\right) \lambda^{l-1}(1+2 C)^{l-1} a_{k-l}+\lambda C \sum_{l=0}^{k-1} \lambda^{l}(1+2 C)^{l} a_{k-l}\right) .
\end{aligned}
$$

Where in the last step we use the induction hypothesis. Now, since $1+C \leq 1+2 C$ the bound recasts as:

$$
E_{2}^{k+1} \leq \lambda\left(\lambda C \sum_{l=1}^{k-1}\left(\begin{array}{c}
l \\
l-1
\end{array}\right) \lambda^{l}(1+2 C)^{l} a_{k-l}+\lambda C \sum_{l=0}^{k-1} \lambda^{l}(1+2 C)^{l} a_{k-l}\right) .
$$


or, after algebraic manipulations,

$$
E_{2}^{k+1} \leq \lambda^{2} C \sum_{l=0}^{k-1}\left(\begin{array}{c}
l+1 \\
l
\end{array}\right) \lambda^{l}(1+2 C)^{l} a_{k-l}
$$

and by setting $\mu=l+1$ we get

$$
E_{2}^{k+1} \leq \lambda^{2} C \sum_{\mu=1}^{(k+1)-1}\left(\begin{array}{c}
\mu \\
\mu-1
\end{array}\right) \lambda^{\mu-1}(1+2 C)^{\mu-1} a_{k+1-\mu}
$$

Lemma 3.4 (Magnitude of the $m$ th extreme). The magnitude of the mth extreme in the kth time step is bounded by

$$
\begin{aligned}
E_{m}^{k} & \leq \lambda^{m} C^{m-1} \sum_{j=1}^{k-1}\left(\begin{array}{c}
k-j \\
k-j-m+1
\end{array}\right) \lambda^{k-j-m+1}(1+2 C)^{k-j-m+1} a_{j} \\
& \stackrel{l=k-j}{=} \lambda^{m} C^{m-1} \sum_{l=m-1}^{k-1}\left(\begin{array}{c}
l \\
l-m+1
\end{array}\right) \lambda^{l-m+1}(1+2 C)^{l-m+1} a_{k-l} .
\end{aligned}
$$

Proof. The proof is the same as in the 2nd extreme and is omitted.

We note that the bounds on the magnitudes of the extremes, i.e., (7), depend on the time step $k$. We next provide bounds for these magnitudes, uniformly with respect to $k$.

Lemma 3.5 (Uniform, with respect to the time step $k$, bound on the extremes). If there exists a constant $M>0$ such that $a_{i} \leq C M$ for every $i \in \mathbb{N}$ and if $\lambda+2 \lambda C<1$, then every extreme $m$ is uniformly, with respect to the time step $k$, bounded as

$$
E_{m}^{k} \leq M\left(\frac{\lambda C}{1-\lambda-2 \lambda C}\right)^{m} .
$$

Proof. At the $k$ th time step, the magnitude of the $m$ th extreme, $m \leq k$, is given by (17):

$$
E_{m}^{k} \leq \lambda^{m} C^{m-1} \sum_{l=m-1}^{k-1}\left(\begin{array}{c}
l \\
l-m+1
\end{array}\right) \lambda^{l-m+1}(1+2 C)^{l-m+1} a_{k-l},
$$

or, since $a_{i} \leq C M$,

$$
\begin{gathered}
E_{m}^{k} \leq \lambda^{m} C^{m} M \sum_{l=m-1}^{k-1}\left(\begin{array}{c}
l \\
l-m+1
\end{array}\right) \lambda^{l-m+1}(1+2 C)^{l-m+1} \\
\stackrel{\nu=l-m+1}{=} \lambda^{m} C^{m} M \sum_{\nu=0}^{k-m}\left(\begin{array}{c}
\nu+m-1 \\
\nu
\end{array}\right) \lambda^{\nu}(1+2 C)^{\nu} .
\end{gathered}
$$

All the terms in the sum are positive; hence the right-hand side is increasing with $k$, and can be bounded for $k=\infty$ as:

$$
E_{m}^{k} \leq \lambda^{m} C^{m} M \sum_{\nu=0}^{\infty}\left(\begin{array}{c}
\nu+m-1 \\
\nu
\end{array}\right) \lambda^{\nu}(1+2 C)^{\nu}=\lambda^{m} C^{m} M \sum_{\nu=0}^{\infty}\left(\begin{array}{c}
\nu+m-1 \\
\nu
\end{array}\right)(\lambda+2 \lambda C)^{\nu} .
$$


We recall that $\sum_{\nu=0}^{\infty}\left(\begin{array}{c}\nu+m-1 \\ \nu\end{array}\right) t^{\nu}=\frac{1}{(1-t)^{m}}$, for $|t|<1$ and since $\lambda+2 \lambda C<1$ we get

$$
E_{m}^{k} \leq \lambda^{m} C^{m} M \frac{1}{(1-\lambda-2 \lambda C)^{m}}=M\left(\frac{\lambda C}{1-\lambda-2 \lambda C}\right)^{m} .
$$

Which proves the assertion of the lemma.

Remark 5. If, moreover, we assume $\lambda+3 \lambda C<1$, instead of $\lambda+2 \lambda C<1$, then $\left\{E_{m}^{k}\right\}$ decreases to 0 with respect to $m$ since $\frac{\lambda C}{1-\lambda-2 \lambda C}<1$, i.e.,

$$
0 \leq E_{m}^{k} \leq M\left(\frac{\lambda C}{1-\lambda-2 \lambda C}\right)^{m} \stackrel{m \rightarrow \infty}{\rightarrow} 0 .
$$

3.3. Variation. In the next theorem we prove that in addition to the magnitude of each extreme separately, also the sum of the extremes is bounded with respect to the time step $k$. This provides with a bound on the total variation increase.

Theorem 3.1 (Total variation increase bound). We assume that requirement (1) and requirement (2) are satisfied for $\lambda$ such that $\lambda+3 \lambda C<1$. Moreover, we assume that $a_{i} \leq C M$, for every $i=1, \ldots, \infty$. Then the sum of the magnitudes of the extremes is uniformly bounded, with respect to the time step $k$, as follows:

$$
\sum_{m=1}^{k} E_{m}^{k} \leq M \frac{1-\lambda-2 \lambda C}{1-\lambda-3 \lambda C}
$$

Moreover, the total variation increase due to the oscillations is bounded:

$$
\mathrm{TVI} \leq 2 M \frac{1-\lambda-2 \lambda C}{1-\lambda-3 \lambda C} .
$$

Proof. At the end of the $k$ th step we have $k$ extremes $E_{1}^{k}, \ldots, E_{k}^{k}$. The sum, with respect to $m$, of their magnitudes can be bounded as

$$
\begin{aligned}
\sum_{m=1}^{k} E_{m}^{k} & \stackrel{\sqrt[8]{\leq}}{\leq} M \sum_{m=1}^{k}\left(\frac{\lambda C}{1-\lambda-2 \lambda C}\right)^{m} \leq M \sum_{m=1}^{\infty}\left(\frac{\lambda C}{1-\lambda-2 \lambda C}\right)^{m} \\
& \leq M \frac{1-\lambda-2 \lambda C}{1-\lambda-3 \lambda C}
\end{aligned}
$$

where the second inequality and the equality are valid since $\lambda+3 \lambda C<1$.

The variation of the oscillatory part, is bounded by twice the magnitude of the extremes, i.e.,

$$
\mathrm{TVI} \leq 2 M \sum_{m=1}^{\infty}\left(\frac{\lambda C}{1-\lambda-2 \lambda C}\right)^{m} \leq 2 M \frac{1-\lambda-2 \lambda C}{1-\lambda-3 \lambda C}
$$

3.4. Variation-revisited. We now follow a different approach that will provide further insight of the pollution process and with a sharper bound on the Total Variation Increase. In this approach we compute the contributions of the increase terms $a_{i}, i=1, \ldots$ in each one of the extremes $E_{m}^{k}$ separately. We subsequently add these contributions with respect to $a_{i}$.

$a_{1}$ contribution. The contribution of $a_{1}$ in the $k$ th step at the $m$ th extreme is given by (77) and reads: $\lambda^{m} C^{m-1}\left(\begin{array}{c}k-1 \\ k-m\end{array}\right) \lambda^{k}(1+2 C)^{k-m}$. Summing these 
contributions with respect to $m$ we end up with the total contribution of $a_{1}$ in the $k$ th time step:

$$
\begin{aligned}
I_{a_{1}}^{k} & =\sum_{m=1}^{k} \lambda^{m} C^{m-1}\left(\begin{array}{c}
k-1 \\
k-m
\end{array}\right) \lambda^{k-m}(1+2 C)^{k-m} a_{1} \\
& \stackrel{\nu=k-m}{=} \lambda^{k} \sum_{\nu=0}^{k-1}\left(\begin{array}{c}
k-1 \\
\nu
\end{array}\right) C^{k-1-\nu}(1+2 C)^{\nu} a_{1} \\
& =\lambda^{k}(1+3 C)^{k-1} a_{1}=\lambda(\lambda+3 \lambda C)^{k-1} a_{1} .
\end{aligned}
$$

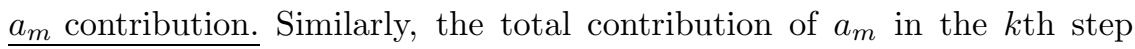
reads:

$$
I_{a_{m}}^{k}=\lambda(\lambda+3 \lambda C)^{k-m} a_{m}
$$

We can now compute the total contribution (of all $a_{i}$ 's) in the $k$ th time step:

$$
I_{\text {tot }}^{k}=\sum_{m=1}^{k} I_{a_{m}}^{k}=\lambda \sum_{m=1}^{k}(\lambda+3 \lambda C)^{k-m} a_{m} .
$$

Corollary 3.1 (Result 1 ). If there exists $M>0$ such that $a_{i} \leq C M$ for every $i \in \mathbb{N}$ and if $\lambda+2 \lambda C<1$, then the total contribution in the $k$ th time step reads:

$$
\mathrm{TVI} \leq \frac{2 \lambda C}{1-(\lambda+3 \lambda C)} M
$$

Proof. Since the increase factors $a_{i}$ are uniformly bounded as $a_{i} \leq C M$, their total contribution, given by (10), reads:

$$
\begin{aligned}
I_{\text {tot }}^{k} & \leq \lambda C M \sum_{m=1}^{k}(\lambda+3 \lambda C)^{k-m} \stackrel{n=\underline{k-m}}{=}=\lambda C M \sum_{n=0}^{k-1}(\lambda+3 \lambda C)^{n} \\
& =\lambda C M \frac{1-(\lambda+3 \lambda C)^{k}}{1-(\lambda+3 \lambda C)} \stackrel{k \rightarrow \infty}{\longrightarrow} \frac{\lambda C}{1-(\lambda+3 \lambda C)} ;
\end{aligned}
$$

hence $I_{\text {tot }}^{\infty}=\lim _{k \rightarrow \infty} I_{\text {tot }}^{k}$ is uniformly, with respect to $k$, bounded. Consequently, the total variation increase due to oscillations is bounded

$$
\mathrm{TVI} \leq 2 \cdot I_{t o t}^{\infty} \leq \frac{2 \lambda C}{1-(\lambda+3 \lambda C)} M
$$

Corollary 3.2 (Result 2). If $a_{i}, i \in \mathbb{N}$ is bounded as $a_{i} \leq C \operatorname{TV}\left(u_{0}\right)$, then (11) reads:

$$
\mathrm{TVI} \leq \frac{2 \lambda C}{1-(\lambda+3 \lambda C)} \mathrm{TV}\left(u_{0}\right)
$$

Corollary 3.3 (Result 3). If, moreover, $\sum_{i=0}^{\infty} a_{i}=A<\infty$, the total variation increase due to oscillations diminishes with respect to the time step $k$.

Proof. We note that the infinite sums $\sum_{i=0}^{\infty}(\lambda+3 \lambda C)^{i}, \sum_{i=0}^{\infty} a_{i}$ converge. Moreover, the sum

$$
\sum_{k=1}^{\infty} I_{\text {tot }}^{k}=\sum_{k=1}^{\infty}\left(\lambda \sum_{j=1}^{k}(\lambda+3 \lambda C)^{k-j} a_{j}\right)=\lambda \sum_{k=1}^{\infty}\left(\sum_{j=1}^{k}(\lambda+3 \lambda C)^{k-j} a_{j}\right),
$$

is the sum of the terms of the Cauchy product of the sums $\sum_{i=1}^{\infty} a_{i}$ and $\sum_{i=1}^{\infty}(\lambda+$ $3 \lambda C)^{i}$. So, the sum $\sum_{k=1}^{\infty} I_{\text {tot }}^{k}<\infty$ also converges; hence $I_{\text {tot }}^{\infty}=\lim _{k \rightarrow \infty} I_{\text {tot }}^{k}=$ 0 . 
Comparison of the two bounds. We have devised two different bounds concerning the Total Variation Increase due to oscillations. The first was immediate summation of the bounds $E_{m}^{k}$ of the magnitudes of the extremes and resulted in the bound (9):

$$
B_{1}=2 M \frac{1-\lambda-2 \lambda C}{1-\lambda-3 \lambda C}
$$

The second one came by investigating the contributions of the increase factors $a_{i}$ and resulted in the bound (11):

$$
B_{2}=\frac{2 \lambda C}{1-\lambda-3 \lambda C} M
$$

We easily see that $\frac{B_{2}}{B_{1}}<1$ for $\lambda+3 \lambda C<1$, and that $\frac{B_{2}}{B_{1}}<\frac{1}{2}$ for $\lambda+4 \lambda C<1$, which means that with a careful selection of the respect factor $\lambda$, the bounds on the increase of the variation in the second approach are improved compared to the first approach.

\section{Main Adaptive Scheme (MAS)}

Moving mesh methods have been employed by several authors in the literature. We refer to [TT03, DZ10, HR10] and the references therein for a thorough presentation of different mathematical approaches and numerical implementations.

For the purpose of this work we shall follow the procedure as studied in AKM01, AMT04, AD06, Arv08, AMS10, Sfa09, where the relocation of the nodes, while keeping their number, is done by studying the "geometric information" of the numerical solution. The basic idea is simple:

"in areas where the numerical solution is smoother/flatter we need

fewer nodes where, in contrary, in areas where the numerical solution is less smooth/flat more nodes are in order"

We start by recalling the Definition 1.1 of MAS and we note that the mesh reconstruction procedure (Step 1) of MAS is performed in each time step. The key point in this procedure is the way that we measure the "geometric information" of the numerical solution. This is accomplished by using two auxiliary functions: the first one - estimator function - measures the geometric information of the numerical solution, while the second one - monitor function-redistributes the nodes according to the information measured by the estimator function. Examples of estimator functions are the arclength estimator, the gradient estimator and the curvature estimator. Throughout this work we shall use the curvature estimator.

The curvature estimator function. We consider a smooth function $U$ and define $K_{U}(x)$ the function that measures the curvature of $U$ :

$$
K_{U}(x)=\frac{\left|U^{\prime \prime}(x)\right|}{\left(1+\left(U^{\prime}(x)\right)^{2}\right)^{3 / 2}} .
$$

Analogously we define the discrete estimator function to compute the curvature of a discrete function

$$
K_{i}^{d s c r}=\frac{\frac{2}{x_{i+1}^{n}-x_{i-1}^{n}}\left|\frac{u_{i}^{n}-u_{i-1}^{n}}{x_{i}^{n}-x_{i-1}^{n}}-\frac{u_{i+1}^{n}-u_{i}^{n}}{x_{i+1}^{n}-x_{i}^{n}}\right|}{\left(\left(1+\left(\frac{u_{i}^{n}-u_{i-1}^{n}}{x_{i}^{n}-x_{i-1}^{n}}\right)^{2}\right)\left(1+\left(\frac{u_{i+1}^{n}-u_{i}^{n}}{x_{i+1}^{n}-x_{i}^{n}}\right)^{2}\right)\left(1+\left(\frac{u_{i+1}^{n}-u_{i-1}^{n}}{x_{i+1}^{n}-x_{i-1}^{n}}\right)^{2}\right)\right)^{1 / 2}},
$$


where $M_{x}^{n}=\left\{x_{i}^{n}, i=1, \ldots, N\right\}$ and $U^{n}=\left\{u_{i}^{n}, i=1, \ldots, N\right\}$ are the non-uniform mesh, and the approximate solution at time step $n$.

By performing a point-by-point evaluation of this discrete estimator, results in a finite sequence that contains the measured information of every node $\left(x_{i}^{n}, u_{i}^{n}\right)$. That is,

$$
K_{U}^{d s c r}=\left\{\left(x_{1}^{n}, K_{1}^{d s c r}\right), \ldots,\left(x_{N}^{n}, K_{N}^{d s c r}\right)\right\} .
$$

Remark 6. In areas where the numerical solution is flat, $K_{i}^{d s c r}=0$; hence no information is extracted. To avoid such a case we select an $\varepsilon>0$ and set $K_{i}^{\text {dscr }}=$ $\max \left\{K_{i}^{d s c r}, \varepsilon\right\}$ for every $i$. A typical value would be $\varepsilon \approx 10^{-14}$ with similar results for larger-but still small—values of $\varepsilon$ (cf. AD06, Arv08).

Remark 7. In the initial condition of a Riemann problem all the nodes (except for the 2 nodes at the top and bottom of the discontinuity) attain the minimum information $K_{i}^{d s c r}=\varepsilon$. The other two nodes attain a very large amount of information. This abrupt change of information yields a non-smooth non-uniform mesh. To avoid such abrupt information change we use a constant $p w>0$ and set $K_{i}^{d s c r}=\left(K_{i}^{d s c r}\right)^{p w}$ for every $i$. This results in a smoother transition of consequent discrete estimator values, a typically value of $p w \approx 0.9$.

Finally, these points, i.e., $\left\{\left(x_{1}^{n}, K_{1}^{d s c r}\right), \ldots,\left(x_{N}^{n}, K_{N}^{d s c r}\right)\right\}$ are interpolated by a continuous piecewise linear function $I_{K^{d s c r}}$.

The monitor function. We first evaluate the monitor function in every old node $x_{i}^{n}$ and then we construct the continuous monitor function by linear interpolation of the discrete monitor values. We integrate the piecewise linear function $I_{K^{d s c r}}$ to find the value of the discrete monitor function in every node $x_{i}^{n}$,

$$
M_{u^{n}}^{x_{i}^{n}}=\int_{0}^{x_{i}^{n}} I_{u^{n}}(x) d x .
$$

This results in a new sequence,

$$
\left.\left\{\left(x_{i}^{n}, M_{u^{n}}^{x_{n}^{n}}\right), i=0, \cdots, N\right)\right\} .
$$

This sequence is positive and strictly increasing since $K_{i}^{d s c r}>0$ for every $i=$ $0, \ldots, N$.

Finally, we interpolate over the values of this sequence by a piecewise linear function $M_{U^{n}}(x)$, which is continuous, positive and strictly increasing and so attains it maximum at the right end $M(1)$. In Figure 7 we present a typical case of an estimator and a monitor function.

Mesh reconstruction. In this step we define a new set of nodes, i.e., $\left\{x_{i}^{n+1}, i=\right.$ $0, \ldots, N\}$ with $x_{0}^{n+1}=0$ that equi-distribute the total information of the monitor function. This is accomplished by solving, recursively and with respect to $x_{i+1}^{n+1}$, the system:

$$
\left\{\begin{array}{l}
x_{0}^{n+1}=0, \\
M_{U^{n}}\left(x_{i+1}^{n+1}\right)-M_{U^{n}}\left(x_{i}^{n+1}\right)=\frac{1}{N} M_{U^{n}}(1), \quad i=0, \ldots, N-1 .
\end{array}\right.
$$

It is easily seen that the complexity of this procedure is $\mathcal{O}(N)$, due to the piecewise linearity of $M_{U^{n}}(x)$. In Figure 8 one can see the affect that the node relocation procedure has on initial condition. 

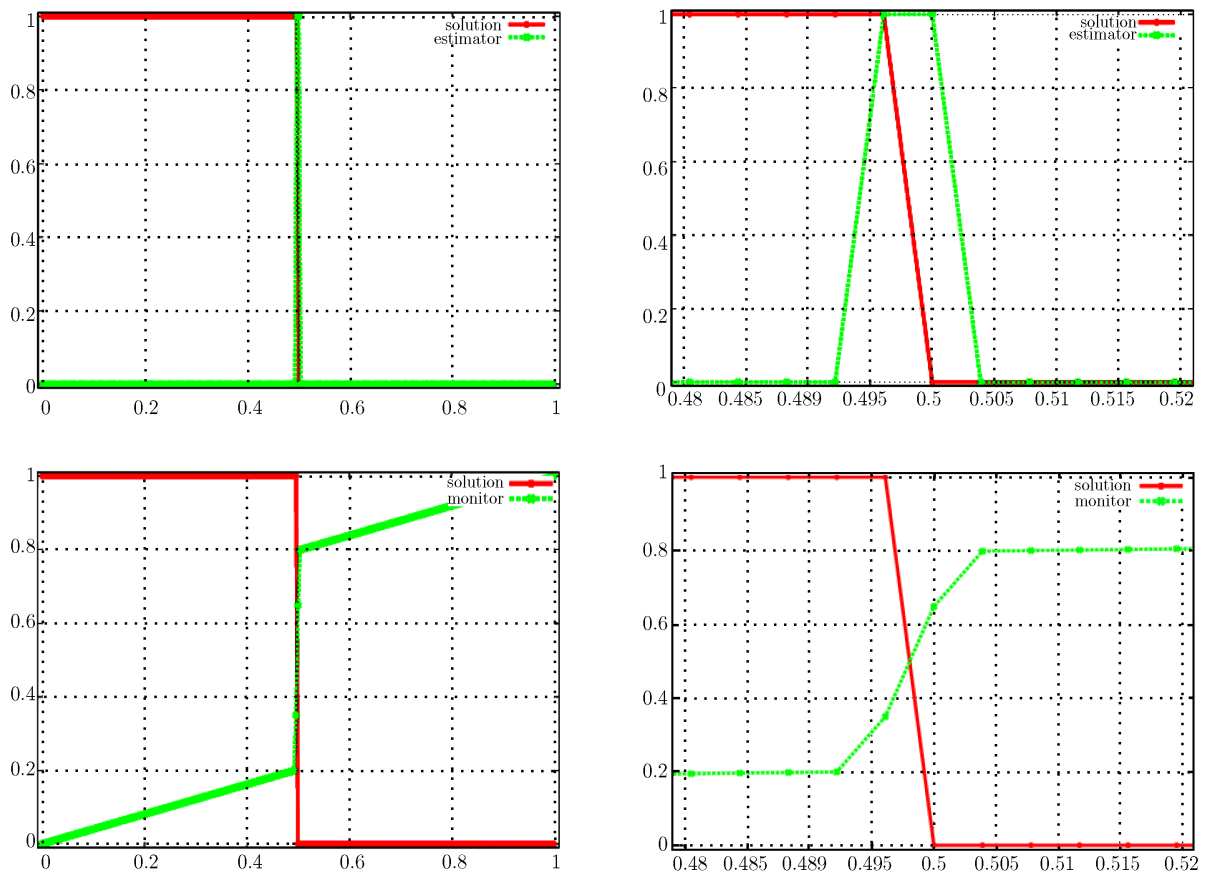

FiguRE 7. A typical estimator (first line) and the corresponding monitor function (second line) depicted along side with the respective numerical solution. The graphs on the right are focused version of the ones on the left.
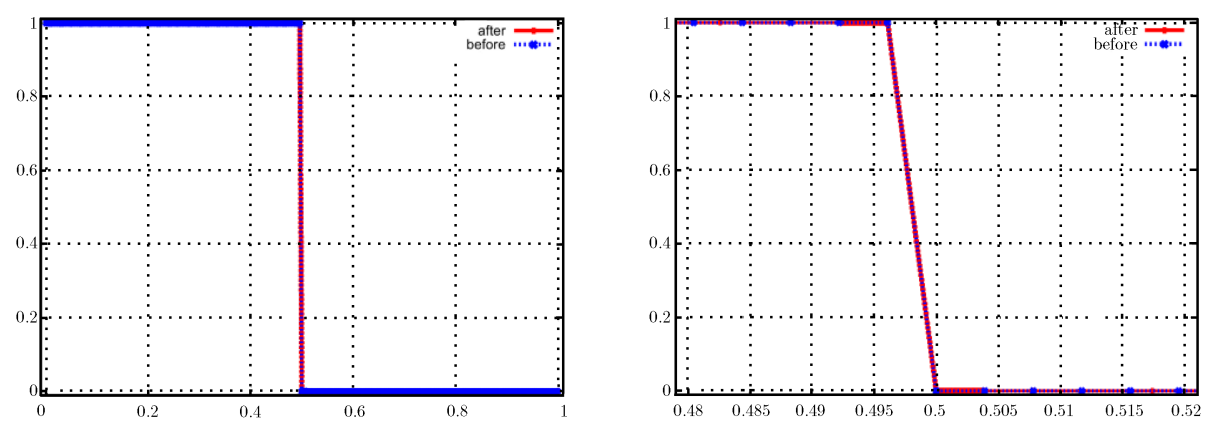

FigURE 8 . We see in this graph the result of the relocation procedure. The density of the nodes is higher around the area of interest of the numerical solution. We also notice that there are nodes placed along the slope of the shock.

For more details on the implementation of the MAS we refer to AKM01, AMT04, AD06, Arv08, Sfa09. 

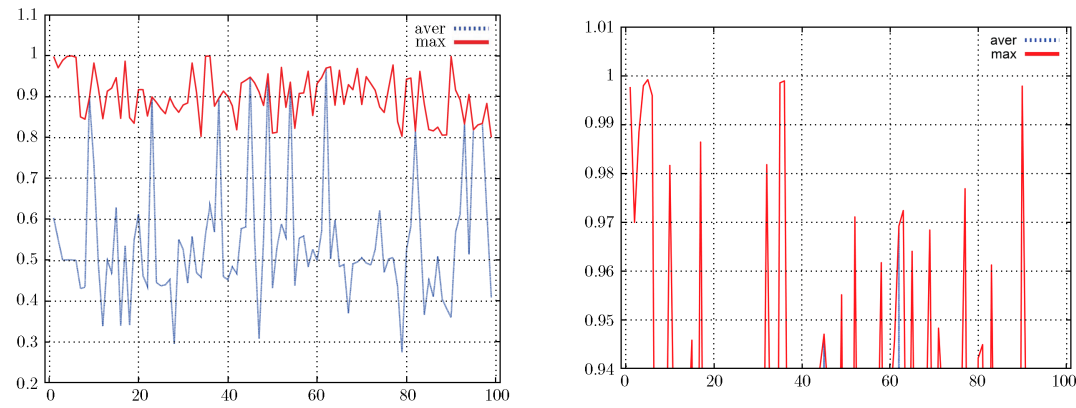

Figure 9. A typical evolution of the maximum and the average $A_{j}, j \in I_{j}^{n}$ with respect to the time steps $n$ (horizontal axis). In the left graph we present the full range of their evolution, while the right graph is a focused version of the left one where one can more clearly see that the value 1 is an upper bound for the $\max _{j \in I_{j}^{n}} A_{j}$, the same is true for the average $A_{j}, j \in I_{j}^{n}$.

\section{Computational COnsiderations}

In this section we discuss the numerical implementation of the coupling requirement (3). We start by defining $I_{j}^{n}$ to be the set of indices of the nodes $x_{j}^{n+1}$ that are placed, after the mesh reconstruction step, "close" to a position of a local extreme of $U^{n}$, that is,

$I_{j}^{n}=\left\{j \mid x_{j}^{n+1} \in\left[x_{i}^{n}, x_{i+1}^{n}\right)\right.$ for some $i$ and $U^{n}$ exhibits local extreme at $x_{i}^{n}$ or $\left.x_{i+1}^{n}\right\}$. For every $j \in I_{j}^{n}, U^{n}$ exhibits a local extreme either at $x_{i}^{n}$ or $x_{i+1}^{n}$, so we set

$$
A_{j}=\frac{x_{i+1}^{n}-x_{j}^{n+1}}{x_{i+1}^{n}-x_{i}^{n}}(1+3 C),
$$

or, respectively,

$$
A_{j}=\frac{x_{j}^{n+1}-x_{i}^{n}}{x_{i+1}^{n}-x_{i}^{n}}(1+3 C),
$$

where the constant $C$ is related to the numerical scheme under discussion. With this notation the coupling requirement reads for the discrete case as:

$$
\max _{j \in I_{j}^{n}} A_{j}<1 .
$$

To impose numerically this requirement we check, for every $j \in I_{j}^{n}$, whether $A_{j} \geq 1$, and if so, we correct accordingly the position of the node $x_{j}^{n+1}$. More specifically, if the local extreme is at the node $x_{i}^{n}$ we set

$$
x_{j}^{n+1}:=x_{j}^{n+1}+\epsilon\left(x_{j}^{n+1}-x_{i}^{n}\right),
$$

where $0<\epsilon<1$, with a typical value of $\epsilon=0.2$.

Similarly, we treat the case where a local extreme is at the node $x_{i+1}^{n}$. We refer to Figure 9 for a typical graph of the maximum and the average $A_{j}, j \in I_{j}^{n}$ with respect to time step $n$. 


\section{NumericAl TESTS}

The numerical schemes that we shall discuss are oscillatory, either due to their dispersive or to their anti-diffusive nature. Here we shall only state their description for non-uniform meshes and prove that they satisfy the evolution requirement (11). We refer to Sfa09 for more details on the derivation the properties and implementation of theses schemes.

We shall deal with the linear transport and the inviscid Burgers equations:

$$
\begin{aligned}
& u_{t}+u_{x}=0, \quad x \in[0,1], \\
& u_{t}+\left(\frac{u^{2}}{2}\right)_{x}=0, \quad x \in[0,1],
\end{aligned}
$$

both with jump initial conditions $u_{0}(x)=\mathcal{X}_{[0,1 / 2]}(x), x \in[0,1]$.

6.1. Richtmyer 2-step Lax-Wendroff. In this approach we consider the nonuniform cell centered discretization of the domain in cells

$$
C_{i}=\left(x_{i-1 / 2}^{n}, x_{i+1 / 2}^{n}\right) \quad \text { with } \quad\left|C_{i}^{n}\right|=h_{i}^{n} .
$$

The mesh $M_{x}^{n}=\left\{x_{i}^{n}, i \in \mathbb{Z}\right\}$ consists of the middle points,

$$
x_{i}^{n}=\frac{x_{i+1 / 2}^{n}+x_{i-1 / 2}^{n}}{2} \quad \text { hence } \quad x_{i}^{n}-x_{i-1}^{n}=\frac{h_{i}^{n}+h_{i-1}^{n}}{2} .
$$

For this description of the grid we propose the following numerical scheme as the generalisation on non-uniform meshes of the Richtmyer 2-step Lax-Wendorff numerical scheme,

$$
\begin{aligned}
u_{i+1 / 2}^{*} & =\frac{h_{i+1}^{n+1}}{h_{i}^{n+1}+h_{i+1}^{n+1}} \hat{u}_{i}^{n}+\frac{h_{i}^{n+1}}{h_{i}^{n+1}+h_{i+1}^{n+1}} \hat{u}_{i+1}^{n}-\frac{\Delta t}{h_{i}^{n+1}+h_{i+1}^{n+1}}\left(f\left(\hat{u}_{i+1}^{n}\right)-f\left(\hat{u}_{i}^{n}\right)\right), \\
u_{i}^{n+1} & =u_{i}^{n}-\frac{\Delta t}{h_{i}}\left(f\left(u_{i+1 / 2}^{*}\right)-f\left(\hat{u}_{i-1 / 2}^{*}\right)\right)
\end{aligned}
$$

or

$$
u_{i}^{n+1}=\hat{u}_{i}^{n}-\frac{\Delta t}{h_{i}^{n+1}}\left(F_{i+1 / 2}-F_{i-1 / 2}\right),
$$

with

$$
\begin{array}{r}
F_{i+1 / 2}=f\left(u_{i+1 / 2}^{*}\right)=f\left(\frac{h_{i+1}^{n+1}}{h_{i}^{n+1}+h_{i+1}^{n+1}} \hat{u}_{i}^{n}+\frac{h_{i}^{n+1}}{h_{i}^{n+1}+h_{i+1}^{n+1}} \hat{u}_{i+1}^{n}\right. \\
\left.-\frac{\Delta t}{h_{i}^{n+1}+h_{i+1}^{n+1}}\left(f\left(\hat{u}_{i+1}^{n}\right)-f\left(\hat{u}_{i}^{n}\right)\right)\right) .
\end{array}
$$

It is straightforward to check that this scheme reduces to the usual Richtmyer 2-step Lax-Wendroff scheme when the mesh is uniform. 
We bound

$$
\begin{aligned}
\left|u_{i}^{n+1}-u_{i}^{n}\right| & \leq \frac{\Delta t}{h_{i}}\left|f\left(u_{i+1 / 2}^{*}\right)-f\left(u_{i-1 / 2}^{*}\right)\right| \leq \frac{\Delta t}{h_{i}} \max \left|f^{\prime}\right|\left|u_{i+1 / 2}^{*}-u_{i-1 / 2}^{*}\right| \\
& \leq \mathrm{CFL}\left|u_{i+1 / 2}^{*}-u_{i-1 / 2}^{*}\right|
\end{aligned}
$$

and

$$
\begin{aligned}
&\left|u_{i+1 / 2}^{*}-u_{i-1 / 2}^{*}\right|=\mid \frac{h_{i+1}^{n+1}}{h_{i}^{n+1}+h_{i+1}^{n+1}} \hat{u}_{i}^{n}+\frac{h_{i}^{n+1}}{h_{i}^{n+1}+h_{i+1}^{n+1}} \hat{u}_{i+1}^{n} \\
&-\frac{\Delta t}{h_{i}^{n+1}+h_{i+1}^{n+1}}\left(f\left(\hat{u}_{i+1}^{n}\right)-f\left(\hat{u}_{i}^{n}\right)\right) \\
&-\frac{h_{i}^{n+1}}{h_{i-1}^{n+1}+h_{i}^{n+1}} \hat{u}_{i-1}^{n}-\frac{h_{i-1}^{n+1}}{h_{i-1}^{n+1}+h_{i}^{n+1}} \hat{u}_{i}^{n} \\
&+\frac{\Delta t}{h_{i-1}^{n+1}+h_{i}^{n+1}}\left(f\left(\hat{u}_{i}^{n}\right)-f\left(\hat{u}_{i-1}^{n}\right)\right) \mid,
\end{aligned}
$$

by setting $\frac{h_{i+1}^{n+1}}{h_{i}^{n+1}+h_{i+1}^{n+1}}=\mu_{1}$ and $\frac{h_{i}^{n+1}}{h_{i-1}^{n+1}+h_{i}^{n+1}}=\mu_{2}$ the previous recasts into:

$$
\begin{aligned}
& \left|u_{i+1 / 2}^{*}-u_{i-1 / 2}^{*}\right|=\mid \mu_{1} \hat{u}_{i}^{n}+\left(1-\mu_{1}\right) \hat{u}_{i+1}^{n}-\frac{\Delta t}{h_{i}^{n+1}+h_{i+1}^{n+1}}\left(f\left(\hat{u}_{i+1}^{n}\right)-f\left(\hat{u}_{i}^{n}\right)\right) \\
& -\mu_{2} \hat{u}_{i-1}^{n}-\left(1-\mu_{2}\right) \hat{u}_{i}^{n}+\frac{\Delta t}{h_{i-1}^{n+1}+h_{i}^{n+1}}\left(f\left(\hat{u}_{i}^{n}\right)-f\left(\hat{u}_{i-1}^{n}\right)\right) \mid \\
& \leq \mu_{1}\left|\hat{u}_{i}^{n}-\hat{u}_{i+1}^{n}\right|+\mu_{2}\left|\hat{u}_{i}^{n}-\hat{u}_{i-1}^{n}\right|+\left|\hat{u}_{i+1}^{n}-\hat{u}_{i}^{n}\right| \\
& +\frac{\Delta t}{2 \min h_{i}^{n+1}} \max \left|f^{\prime}\right|\left|\hat{u}_{i+1}^{n}-\hat{u}_{i}^{n}\right|+\frac{\Delta t}{2 \min h_{i}^{n+1}} \max \left|f^{\prime}\right|\left|\hat{u}_{i}^{n}-\hat{u}_{i-1}^{n}\right| \\
& \leq\left(1+\mu_{1}+\mu_{2}+\mathrm{CFL}\right) \max \left\{\left|\hat{u}_{i-1}^{n}-\hat{u}_{i}^{n}\right|,\left|\hat{u}_{i}^{n}-\hat{u}_{i+1}^{n}\right|\right\} \\
& \leq(3+\mathrm{CFL}) \max \left\{\left|\hat{u}_{i-1}^{n}-\hat{u}_{i}^{n}\right|,\left|\hat{u}_{i}^{n}-\hat{u}_{i+1}^{n}\right|\right\} \text {. }
\end{aligned}
$$

where the last inequality is valid since $0<\mu_{1}, \mu_{2} \leq 1$. So the overall bound reads,

$$
\left|u_{i}^{n+1}-\hat{u}_{i}^{n}\right| \leq \operatorname{CFL}(3+\mathrm{CFL}) \max \left\{\left|\hat{u}_{i+1}^{n}-\hat{u}_{i}^{n}\right|,\left|\hat{u}_{i}^{n}-\hat{u}_{i-1}^{n}\right|\right\} .
$$

The constant $C$ in this case is chosen to be $C=\mathrm{CFL}(3+\mathrm{CFL})$, for this choice the evolution requirement is satisfied. We refer to Figures 10 and 11 for comparative graphs between the uniform and non-uniform mesh case for the Richtmyer 2-step Lax-Wendroff scheme. 

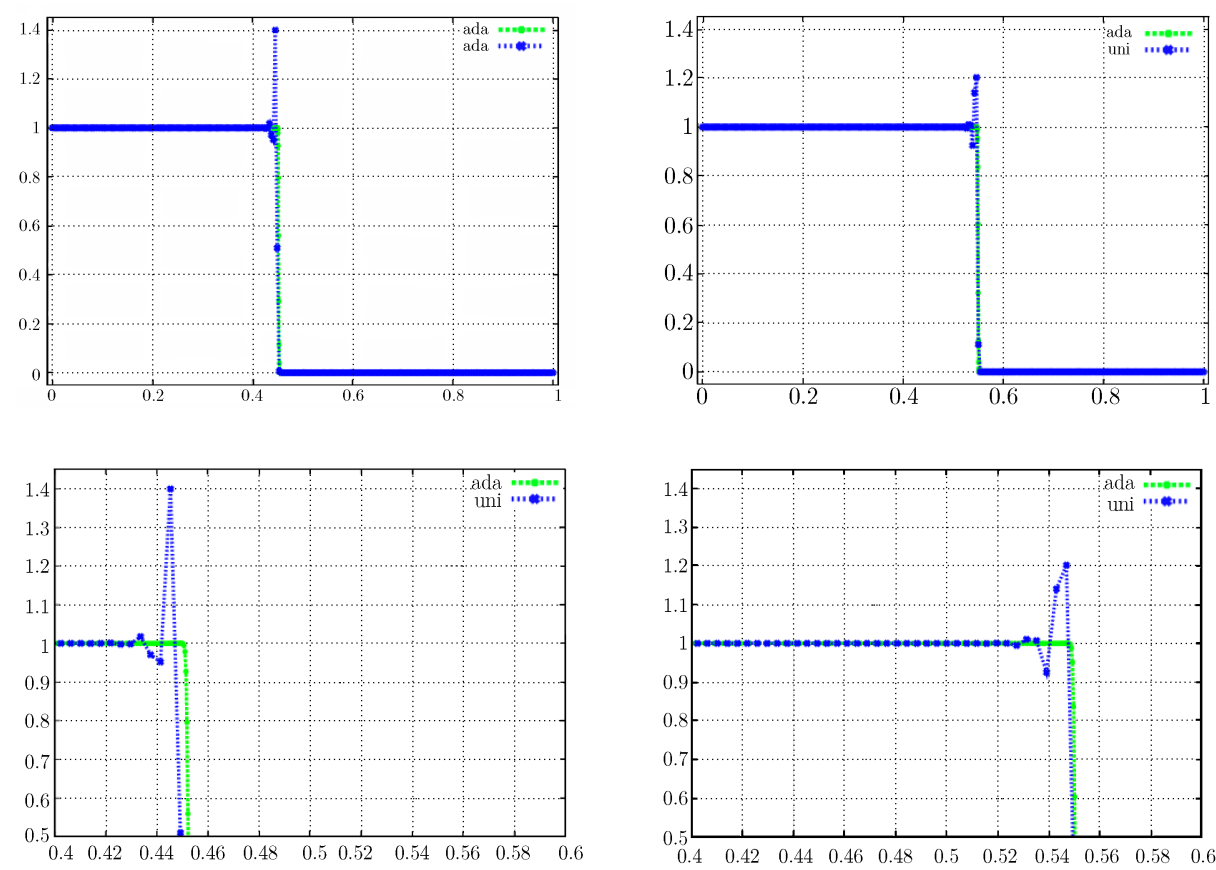

FIgURE 10. Inviscid Burgers equation using the Richtmyer 2-step Lax-Wendroff over uniform and non-uniform meshes. One time step per column, full and focused domain per row. The uniform mesh case exhibits oscillations due to the dispersive nature of the numerical scheme where as the non-uniform mesh case is clean.

6.2. MacCormack. For the same description of the grid as in Section6.1 we study the following scheme as a non-uniform mesh generalization of the MacCormack scheme,

$$
\begin{aligned}
u_{i}^{*} & =\hat{u}_{i}^{n}-\frac{2 \Delta t}{h_{i}^{n+1}+h_{i+1}^{n+1}}\left(f\left(\hat{u}_{i+1}^{n}\right)-f\left(\hat{u}_{i}^{n}\right)\right), \\
u_{i}^{* *} & =u_{i}^{*}-\frac{2 \Delta t}{h_{i-1}+h_{i}}\left(f\left(u_{i}^{*}\right)-f\left(u_{i-1}^{*}\right)\right), \\
u_{i}^{n+1} & =\frac{\hat{u}_{i}^{n}+u_{i}^{* *}}{2} .
\end{aligned}
$$

We rewrite the scheme in the following form, for $f_{i}^{*}=f\left(u_{i}^{*}\right)$ and $f_{i}=f\left(\hat{u}_{i}^{n}\right)$

$$
\begin{aligned}
u_{i}^{n+1} & =\frac{\hat{u}_{i}^{n}}{2}+\frac{u_{i}^{*}-\frac{2 \Delta t}{h_{i-1}+h_{i}}\left(f_{i}^{*}-f_{i-1}^{*}\right)}{2} \\
& =\frac{\hat{u}_{i}^{n}}{2}+\frac{\hat{u}_{i}^{n}-\frac{2 \Delta t}{h_{i}^{n+1}+h_{i+1}^{n+1}}\left(f_{i+1}-f_{i}\right)-\frac{2 \Delta t}{h_{i-1}^{n+1}+h_{i}^{n+1}}\left(f_{i}^{*}-f_{i-1}^{*}\right)}{2} \\
& =\hat{u}_{i}^{n}-\frac{\Delta t}{h_{i}^{n+1}+h_{i+1}^{n+1}}\left(f_{i+1}-f_{i}\right)-\frac{\Delta t}{h_{i-1}^{n+1}+h_{i}^{n+1}}\left(f_{i}^{*}-f_{i-1}^{*}\right) .
\end{aligned}
$$



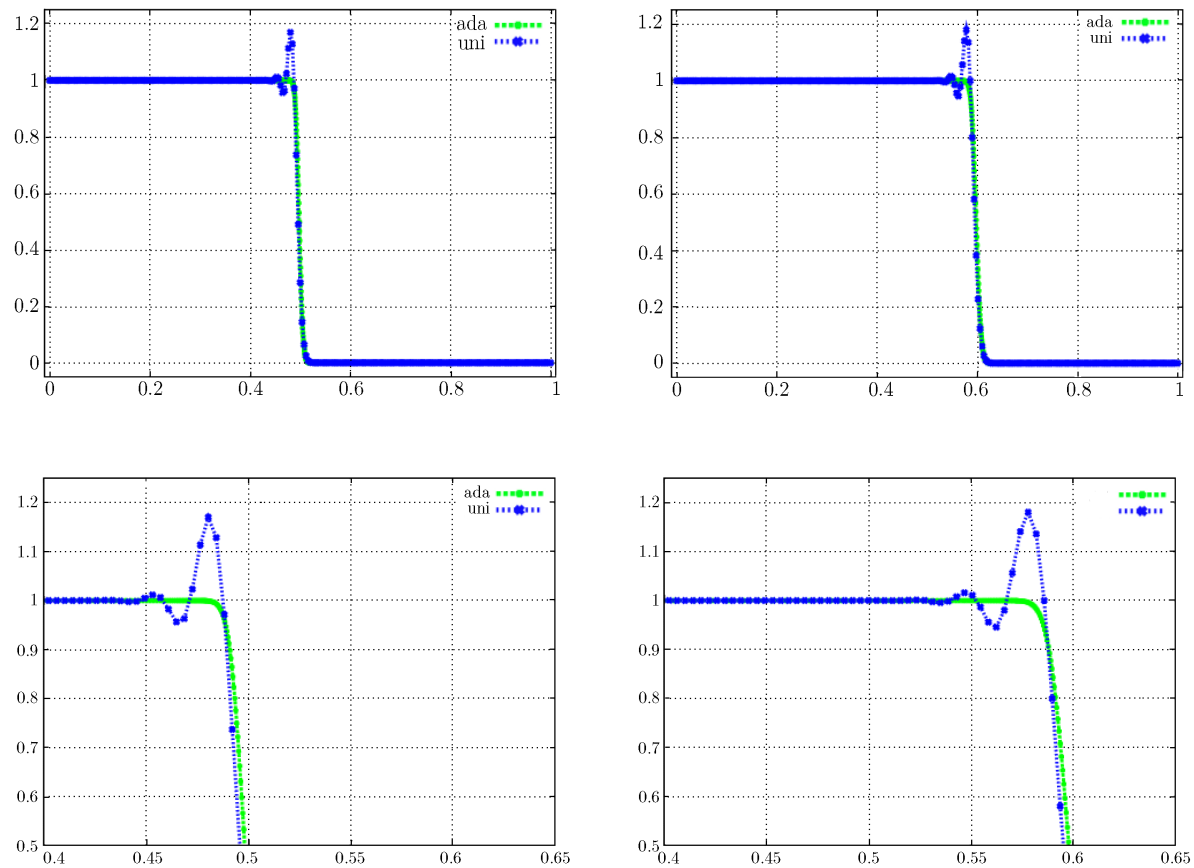

FIgURE 11. Transport equation with velocity $a=1$, using the Richtmyer 2-step Lax-Wendroff. Again, oscillations appear in the uniform mesh case, whereas the non-uniform one is clean.

To prove the evolution requirement (1) for this scheme we need to bound

$$
\begin{aligned}
\mid u_{i}^{n+1} & -\hat{u}_{i}^{n}|=|-\frac{\Delta t}{h_{i}^{n+1}+h_{i+1}^{n+1}}\left(f_{i+1}-f_{i}\right)-\frac{\Delta t}{h_{i-1}^{n+1}+h_{i}^{n+1}}\left(f_{i}^{*}-f_{i-1}^{*}\right) \mid \\
& \leq \frac{\mathrm{CFL}}{2}\left(\left|\hat{u}_{i+1}^{n}-\hat{u}_{i}^{n}\right|+\left|u_{i}^{*}-u_{i-1}^{*}\right|\right) \\
& \leq \frac{\mathrm{CFL}}{2}\left(\left|\hat{u}_{i+1}^{n}-\hat{u}_{i}^{n}\right|+\left|\hat{u}_{i}^{n}-\hat{u}_{i-1}^{n}\right|+\mathrm{CFL}\left|\hat{u}_{i}^{n}-\hat{u}_{i-1}^{n}\right|+\mathrm{CFL}\left|\hat{u}_{i+1}^{n}-\hat{u}_{i}^{n}\right|\right) \\
& \leq \mathrm{CFL}(1+\mathrm{CFL}) \max \left\{\left|\hat{u}_{i+1}^{n}-\hat{u}_{i}^{n}\right|,\left|\hat{u}_{i}^{n}-\hat{u}_{i-1}^{n}\right|\right\} .
\end{aligned}
$$

So, the constant $C$ in this case is chosen to be $C=\mathrm{CFL}(1+\mathrm{CFL})$ and for this choice the Evolution Requirement is satisfied. We refer to Figure 12 for a comparison graph between the uniform and non-uniform mesh case for the MacCormack scheme.

6.3. Unstable Centered-FTCS. In this approach we consider the non-uniform mesh

$$
M_{x}^{n}=\left\{x_{i}^{n}, i \in \mathbb{Z}\right\} \quad \text { with } \quad h_{i}^{n}=x_{i}^{n}-x_{i-1}^{n} .
$$

The middle points $x_{i-1 / 2}^{n}=\frac{x_{i-1}^{n}+x_{i}^{n}}{2}$ define a partition of the domain in cells,

$$
C_{i}^{n}=\left(x_{i-1 / 2}^{n}, x_{i+1 / 2}^{n}\right) \quad \text { with } \quad\left|C_{i}^{n}\right|=\frac{h_{i}^{n}+h_{i+1}^{n}}{2} .
$$

For this description of the grid we discuss the known to be unstable Forward in Time Centered in Space (FTCS) scheme. The instability of this scheme and the 

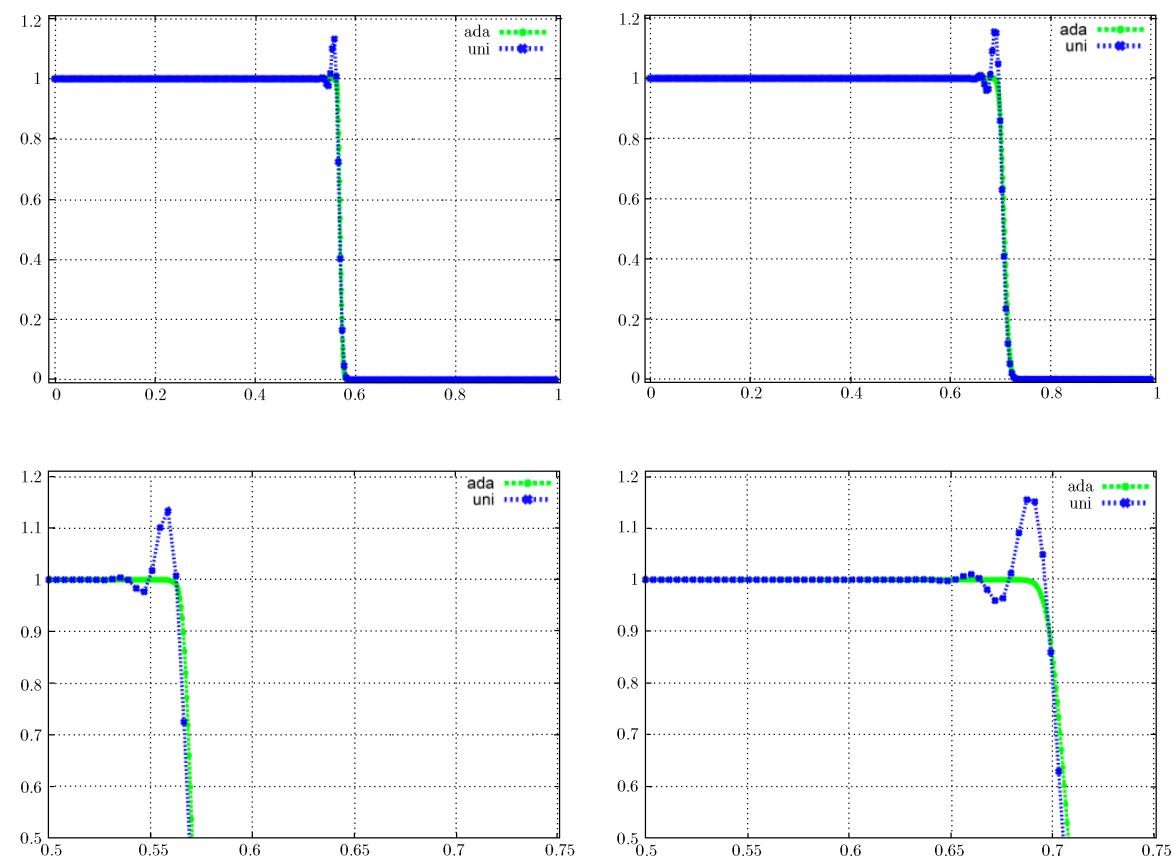

FiguRE 12. Transport equation with velocity $a=1$, using the MacCormack scheme over uniform and non-uniform meshes. Again, oscillations are apparent in the uniform mesh case, due to the dispersive nature of the scheme, whereas the non-uniform is clean.

oscillations that it presents are due to its anti-diffusive nature

$$
u_{i}^{n+1}=\hat{u}_{i}^{n}-\frac{\Delta t}{h_{i}^{n+1}+h_{i+1}^{n+1}}\left(f\left(\hat{u}_{i+1}^{n}\right)-f\left(\hat{u}_{i-1}^{n}\right)\right) .
$$

This scheme can be written in conservative form as follows:

$$
u_{i}^{n+1}=\hat{u}_{i}^{n}-\frac{2 \Delta t}{h_{i}^{n+1}+h_{i+1}^{n+1}}\left(F_{i+1 / 2}^{n}-F_{i-1 / 2}^{n}\right),
$$

with

$$
F_{i+1 / 2}^{n}=\frac{f\left(\hat{u}_{i}^{n}\right)+f\left(\hat{u}_{i+1}^{n}\right)}{2}
$$

We deduce easily that

$$
\begin{aligned}
\left|u_{i}^{n+1}-\hat{u}_{i}^{n}\right| & \leq \frac{\Delta t}{2 \min h_{i}^{n+1}} \max \left|f^{\prime}\right|\left|\hat{u}_{i+1}^{n}-\hat{u}_{i-1}^{n}\right| \leq \frac{\mathrm{CFL}}{2}\left(\left|\hat{u}_{i+1}^{n}-\hat{u}_{i}^{n}\right|+\left|\hat{u}_{i}^{n}-\hat{u}_{i-1}^{n}\right|\right) \\
& \leq \mathrm{CFL} \max \left\{\left|\hat{u}_{i+1}^{n}-\hat{u}_{i}^{n}\right|,\left|\hat{u}_{i}^{n}-\hat{u}_{i-1}^{n}\right|\right\} .
\end{aligned}
$$

The constant $C$ in this case is chosen to be $C=\mathrm{CFL}$, for this choice the Evolution requirement is satisfied. We refer to Figure 13 for a comparison graph between the uniform and non-uniform mesh case for the FTCS scheme. 

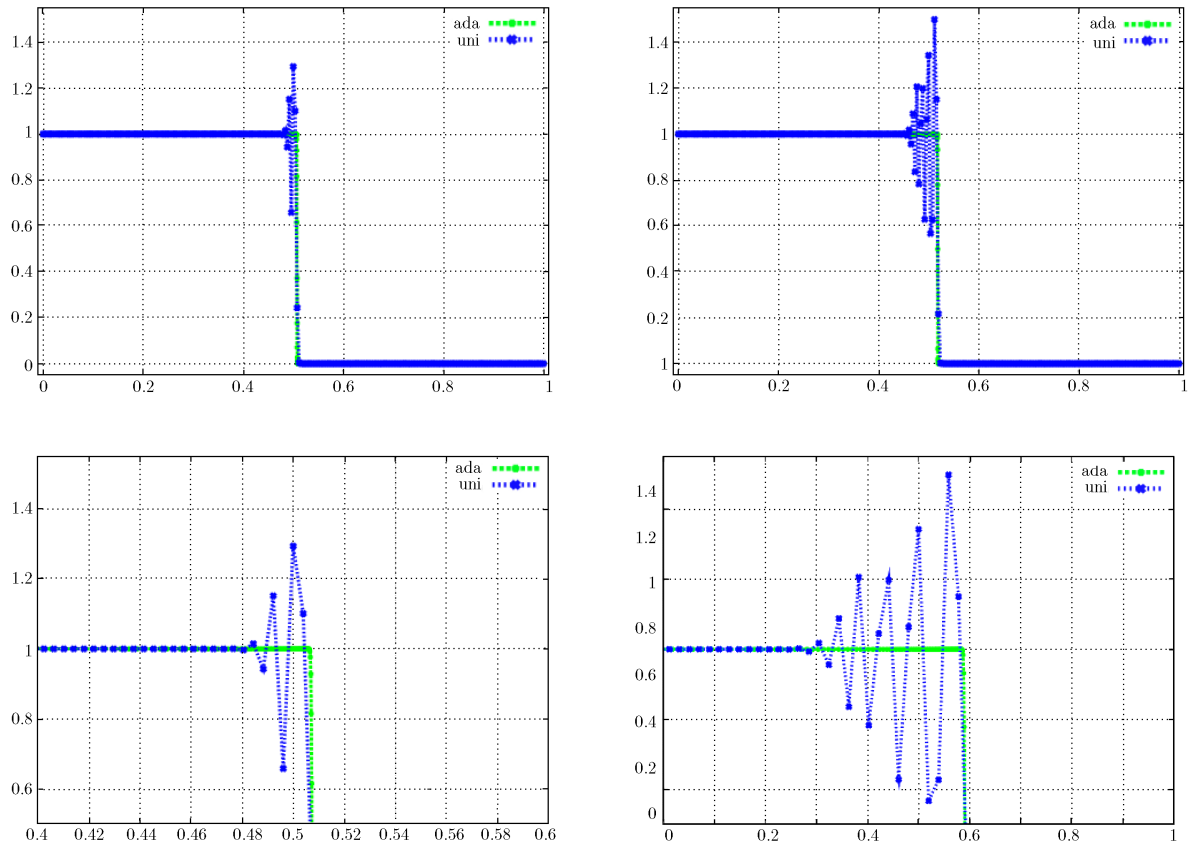

Figure 13. Inviscid Burgers equation, using the unstable FTCS. The oscillations in the uniform case are due to the anti-diffusive nature of the numerical scheme. The non-uniform mesh case is clean.

\section{Conclusions}

In this work we have investigated the creation and evolution of oscillations that numerical solutions present over non-uniform, adaptively redefined meshes. The mesh reconstruction is driven by the geometry of the numerical solution itself and the solution update is performed by interpolation over piecewise linear functions. The numerical schemes we consider are 3-point non-uniform versions of oscillatory numerical schemes. The overall process is driven by the Main Adaptive Scheme (MAS).

We prove under specific assumptions/requirements on a) the mesh reconstruction, b) the solution update over the new mesh, and c) the numerical schemes that the numerical solution is of Bounded Total Variation; furthermore, under more strict assumptions, we prove that the increase of the Total Variation decreases with time. We provide numerical tests that exhibit the stabilization properties of the MAS and support the analytical results of this work.

\section{REFERENCES}

[AD06] Ch. Arvanitis and A. I. Delis, Behavior of finite volume schemes for hyperbolic conservation laws on adaptive redistributed spatial grids, SIAM J. Sci. Comput. 28 (2006), 1927-1956. MR2272195 (2007m:65064)

[AKM01] Ch. Arvanitis, Th. Katsaounis, and Ch. Makridakis, Adaptive finite element relaxation schemes for hyperbolic conservation laws, Math. Model. Anal. Numer. 35 (2001), 17-33. MR.1811979 (2002g:65119) 
[AMS10] Ch. Arvanitis, Ch. Makridakis, and N. Sfakianakis, Entropy conservative schemes and adaptive mesh selection for hyperbolic conservation laws, J. Hyp. Diff. Eq. 3 (2010), 383-404. MR2735815 (2011k:65128)

[AMT04] Ch. Arvanitis, Ch. Makridakis, and A. Tzavaras, Stability and convergence of a class of finite element schemes for hyperbolic systems of conservation laws, SIAM J. Numer. Anal. 42 (2004), 1357-1393. MR2114282(2005m:65204)

[Arv08] Ch. Arvanitis, Mesh redistribution strategies and finite element method schemes for hyperbolic conservation laws, J. Sci. Computing 34 (2008), 1-25. MR2367009 (2008j:65154)

[DD87] E. Dorfi and L. Drury, Simple adaptive grids for 1d initial value problems, J. Computational Physics 69 (1987), 175-195.

[DZ10] A. van Dam and P. A. Zegeling, Balanced monitoring of flow phenomena in moving mesh methods, Commun. Comput. Physics 7 (2010), 138-170. MR2673131 (2011g:65157)

[For88] B. Fornberg, Generation of finite difference formulas on arbitrary spaced grids, Mathematics of Computations 51 (1988), 699-706. MR.935077 (89b:65055)

[HH83] A. Harten and J. Hyman, Self adjusting grid methods for one-dimensional hyperbolic conservation laws, J. Comput. Physics 50 (1983), 235-269. MR707200 (85g:65111)

[HR10] W. Huang and R.D. Russel, Adaptive moving mesh methods, Springer, 2010. MR2722625

[Kro97] D. Kroener, Numerical schemes for conservation laws, Wiley Teubner, 1997. MR1437144 (98b:65003)

[LeV92] R. LeVeque, Numerical methods for conservation laws, second ed., Birkhäuser Verlag, 1992. MR1153252 (92m:65106)

[LeV02] _ Finite volume methods for hyperbolic problems, first ed., Cambridge Texts in Applied Mathematics, 2002. MR1925043(2003h:65001)

[LR56] P. D. Lax and R. Richtmyer, Survey of the stability of linear finite difference equations, Comm. Pure Appl. Math. 9 (1956), 267-293. MR0079204 (18:48c)

[Luc85] B. J. Lucier, A stable adaptive numerical scheme for hyperbolic conservation laws, SIAM J. Num. Anal. 22 (1985), 180-203. MR772891 (86d:65123)

[Luc86] - A moving mesh numerical method for hyperbolic conservation laws, Math. Comput. 46 (1986), no. 173, 59-69. MR815831 (87m:65141)

[LW60] P. D. Lax and B. Wendroff, Systems of conservation laws, Comm. Pure Appl. Math. 13 (1960), 217-237. MR0120774 (22:11523)

[Sfa09] N. Sfakianakis, Finite difference schemes on non-uniform meshes for hyperbolic conservation laws, Ph.D. thesis, University of Crete, 2009.

[TT03] H. Tang and T. Tang, Adaptive mesh methods for one- and two-dimensional hyperbolic conservation laws, SIAM J. Numerical Analysis 41 (2003), 487-515. MR2004185 (2004f:65143)

University OF VIENNA, Austria

Current address: Johannes Gutenberg University, Mainz, Germany

E-mail address: sfakiana@uni-mainz.de 